\title{
Searching for Planets with the Space Interferometry Mission
}

\author{
Stephen Unwin \\ Jet Propulsion Laboratory, California Institute of Technology, 4800 Oak Grove \\ Drive, Pasadena, CA 91109, USA
}

\begin{abstract}
The Space Interferometry Mission (SIM) will be the first space-based long baseline Michelson interferometer designed for precision astrometry. It will address a wide range of problems in stellar astrophysics and Galactic structure, delivering precision astrometry of stars down to 20 magnitude throughout the entire Galaxy. SIM uses a 10-m Michelson interferometer in Earth-trailing solar orbit to provide 4 microarcsecond ( $\mu$ as) precision astrometry. With a 5-year mission lifetime, SIM will be a powerful tool for discovering planets around nearby stars, through detection of the stellar reflex motion. The astrometric method complements the radial velocity technique which as already yielded many new planets, with an important benefit of directly measuring planetary masses. SIM will have a single-measurement precision of 1 mas in a frame defined by nearby reference stars, enabling searches for planets with masses as small as a few earth masses around the nearest stars. More massive planets will be detectable to much larger distances. In addition to precision astrometry SIM will also serve an important role as a technology precursor for future astrophysics missions using interferometers. Two technologies demonstrated . will be high dynamic-range aperture synthesis imaging at 10-milliarcsec resolution in the optical, and fringe nulling to $10^{-4}$.
\end{abstract}

\section{Introduction}

The Space Interferometry Mission (SIM) is designed as a space-based optical interferometer operating in the visible waveband. This mission will open up many areas of astrophysics, via astrometry with unprecedented accuracy during its 5-year mission. In its wide-angle mode, SIM will be capable to provide a 4 uas precision absolute position measurements of stars, with parallaxes to comparable accuracy [1]. The expected proper motion accuracy is around $2 \mu \mathrm{ss} / \mathrm{yr}$, corresponding to a transverse velocity of $10 \mathrm{~m} / \mathrm{s}$ at a distance of $1 \mathrm{kpc}$. It is expected to achieve an accuracy of 1 pas in one hour, over a narrow field of view. In this mode, SIM will search for planetary companions to nearby stars, by detecting the astrometric 'wobble' relative to nearby reference stars.

The SIM architecture comprises three 10-m baseline Michelson interferometers with parallel baselines. Collectors at each end of the structure contain afocal telescopes which compress the input beam from a diameter of $0.3 \mathrm{~m}$. Two telescope pairs observe bright guide stars, to stabilize the optical system, and a third pair observes the science target. A fourth set of optics are 
carried for redundancy. Light from each pair is directed to a beam combiner through as series of fold mirrors. One light path passes through a movable delay line which tracks the white-light fringe. The position of this delay line is the fundamental astrometric quantity measured by SIM. An internal metrology beam is used to measure the path difference between the two arms of the interferometer. The SIM Project is currently evaluating two designs for the layout of the three interferometers. In one of the designs, a deployable metrology boom measures the positions of the individual collectors, and the baseline lengths are triangulated. In the alternate design, the three baselines are coincident, and a single metrology beam measures the baseline length; this design has a more complicated optical layout, but a simpler metrology system.

The telescope field of regard is $15^{\circ}$; this is the sky area accessible while the instrument is inertially pointed. Astrometry is performed by measuring the change in white-light fringe delay between successive targets. Global astrometry is accomplished by 'tiling' the sky with grid stars which serve as positional references for the astrometry. A summary of the key instrument and mission parameters is given in Table 1.

While SIM is primarily an astrometric instrument, it will serve as a technology pathfinder for future astrophysics missions, such as the Terrestrial Planet Finder (TPF). Key technologies needed by future missions will be demonstrated by SIM, then carried over directly or readily adapted. For TPF the nulling beam combiner, which operates by applying a polarization flip to one arm of the interferometer will demonstrate a null depth (suppression of on-axis light) to $10^{-4}$. A scientific goal of this observing mode for SIM is imaging of reflected light from dust disks around main-sequence stars. Nulling mode greatly increases the effective sensitivity to a disk, by canceling most of the light from the bright central star.

SIM will also operate as an imaging instrument, using aperture synthesis to provide a fully diffraction-limited aperture of $10 \mathrm{~m}$ in the optical band. The image resolution will be an unprecedented 10 milliarcsec (mas) in the optical. High dynamic range images will be possible because of the instrument stability and accurate fringe calibration. An example of an imaging target for SIM is a nearby active galactic nucleus with strong $\mathrm{H} \alpha$ line emission near its center. By imaging the line-emitting gas, SIM will measure the mass distribution in the very dense central region.

SIM is a key element of the NASA Origins Program. The long-range goal of this program is to seek answers to questions of the origins of galaxies, stars, and planets, and ultimately life itself. The program has a phased approach to the technical challenges of the proposed space missions. More information on SIM and Origins is available on the Web at: http://sim.jpl.nasa.gov. 
Table 1. SIM Instrument and Mission Parameters

\begin{tabular}{ll}
\hline \hline Baseline & $10 \mathrm{~m}$ \\
Wavelength range & $0.4-1.0 \mu \mathrm{m}$ \\
Telescope aperture & $0.3 \mathrm{~m}$ diameter \\
Astrometric Field of Regard & $15^{\circ}$ \\
Imaging field of view & $0.3 \mathrm{arcsec}$ \\
Detector & Si CCD \\
Orbit & Earth-Trailing solar orbit \\
Mission Duration & 5 years (launch June, 2005) \\
Astrometry (wide-angle) & 4 mas mission accuracy \\
Astrometric Sensitivity & 20 mag in 4 hours \\
Astrometry (narrow-angle) & $1 \mu$ as in 1 hour \\
Imaging Resolution & 10 milliarcsec \\
Imaging Sensitivity (point source) 25 mag in 1 hour \\
Interferometric Nulling & Null depth $10^{-4}$ \\
\hline
\end{tabular}

\section{Nearby Planetary Systems}

The discovery of planets around other stars [2], [3] has brought into focus a long standing, but poorly understood problem in astronomy, namely the formation of planetary systems. These new data are the first solid pieces in a puzzle which will take some time to unravel. SIM will contribute strongly to the observational database available for testing of solar-system formation theories, by searching for planets. It will be capable of detecting planets down to Earth mass around the nearest stars, and Jupiter-mass planets out to a kiloparsec.

An important goal for the mission is to detect the presence of earthsized planets orbiting nearby solar-type stars and study their characteristics in order to learn about the formation and evolution of planetary systems that might include habitable planets. SIM will search for earth-sized planets orbiting in or near the habitable zones of 50 of the most suitable solar-type stars.

Because planets are extremely faint compared to the star around which they orbit - typically $10^{9}$ times fainter in the visible waveband, indirect detection techniques must be used. In a two-body system, the components orbit around the center of mass of the system, so that the presence of a comparatively small body, such as a planet, around a star induces a 'wobble' on its motion which can be detected by tracing the motion of the center of light of the system. The well-known radial velocity method utilizes the Doppler shift resulting from the radial motion of the star. With a resolution of better than $10 \mathrm{~m} / \mathrm{s}$, many giant planets around several solar-type stars have been found [3]. With this technique, the mass of the planet is unknown to a factor $(\sin i)$ where $i$ is the inclination of orbit plane to the line of sight. 


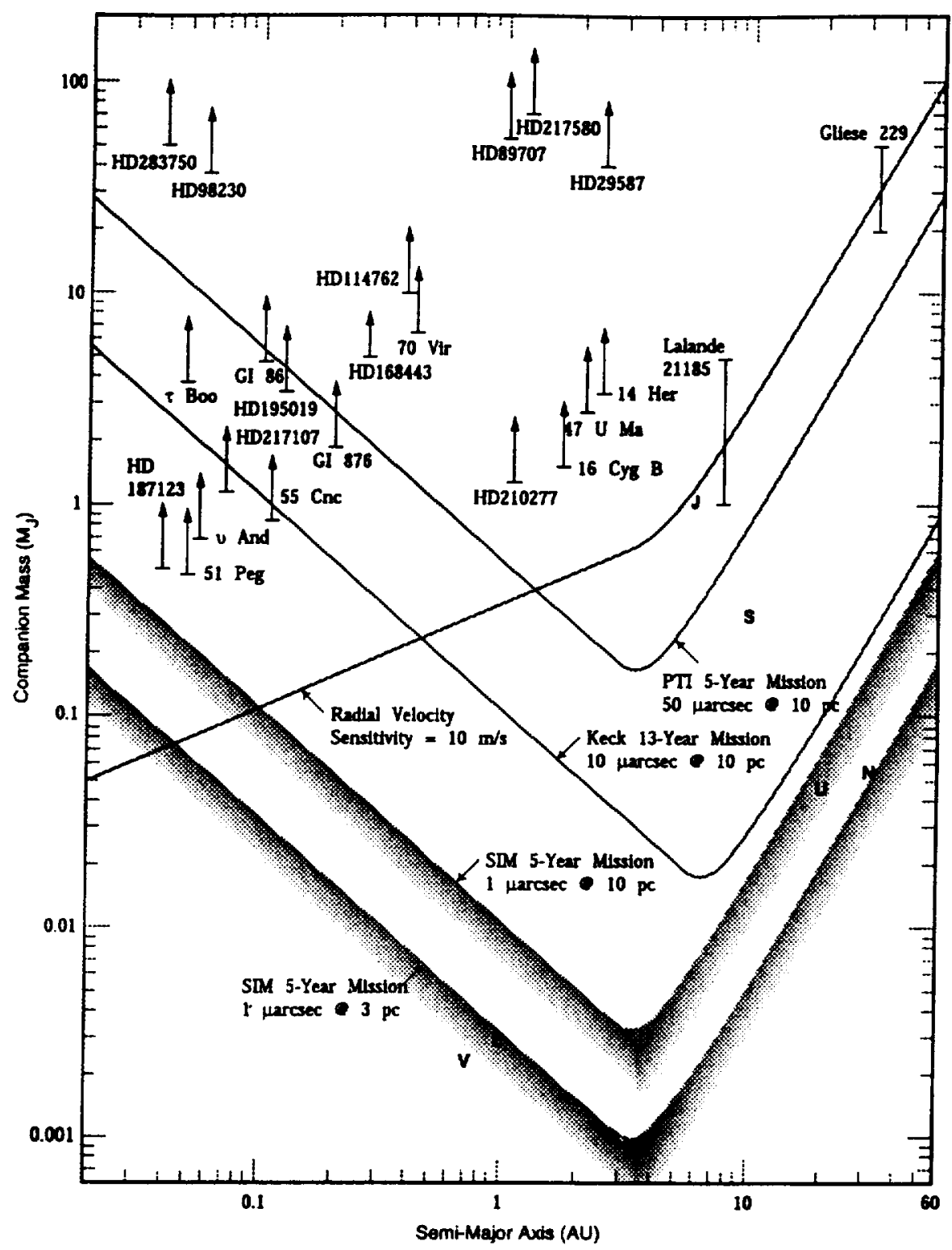

Fig. 1. Masses and orbital semi-major axes for known substellar objects. Planets in our own solar system are also shown. Diagonal lines represent the detection sensitivities of the several instruments: radial velocity with a $10 \mathrm{~m} / \mathrm{s}$ sensitivity; Palomar Testbed Interferometer and Keck Interferometer for stars at $10 \mathrm{pc}$; and SIM. For SIM, the curves are for stars at $3 \mathrm{pc}$ and $10 \mathrm{pc}$ distance. Note the reduced sensitivity of all instruments to very long-period orbits 
Figure 1 shows where the known planets (including our own solar system) lie in the mass - orbit-radius plane, together with the sensitivity limits of the two main detection methods.

SIM detects the the transverse reflex motion of the target star with respect to a reference frame of nearby $\left(\leq 1^{\circ}\right)$ stars. Our own Sun would reveal the presence of its planetary system to an observer at 10 parsec away with a signal of \pm 500 ras. The dominant sources of the wobble are Jupiter and Saturn, and these would be easy SIM targets. In its narrow-angle mode, SIM will reach a precision of about 1 tas in a 1-hour measurement. Repeated visits during the mission will allow the characterization of orbits with signatures smaller than this value. This is of the same order as the reflex motion induced by an Earth-mass planet around one of the nearest few G-type stars.

The recent announcement [4] of a triple planetary system around Upsilon Andromedae provides additional impetus for planet searching for SIM. Now that a multiple system has been found, one can begin asking more detailed questions of planetary searches. What range of orbit periods and masses are typical? Are the orbits circular? Are they coplanar? Continued radial velocity searches will provide many answers, and probably several new multiplanet systems. SIM has at least two unique contributions to make. It can measure all the astrometric parameters, and test the coplanarity of the orbits, which cannot be done with radial velocities. And it will reach much lower in mass than the radial velocity method, exploring the Earth-Uranus mass range for a large number of systems. Unlike Hipparcos, and all the proposed future astrometry missions, SIM is a pointed instrument. This capability will allow SIM to perform very detailed studies of the most interesting planetary systems.

SIM will detect large (5 to 10 earth masses) terrestrial planets orbiting nearby solar-type stars and study their characteristics in order to learn about the role of rocky cores in the formation and evolution of planetary systems. It will search a large nearby sample for planets which formed as rocky cores in the region closer to their parent stars than the inner boundary of the ice condensation zones in their proto-planetary disks.

SIM will explore the prevalence of planetary systems as a function of stellar type along the main sequence, since the spectral characteristics of the parent star do not affect the sensitivity of the astrometric detection method.

\section{SIM Astrometric Science}

The discovery and characterization of planetary systems around nearby stars is a prime objective for SIM, as indicated above. But the science program for SIM is very broad, and will impact many areas of astrophysics including fundamental astrophysics, the cosmic distance scale, and Galactic dynamics and structure [5]. SIM offers the ability to make astrometric measurements with an accuracy far in excess of ground-based observations, or any other 
space mission in the near term. As well as contributing strongly to known astrophysical problems, it has the potential for completely unexpected results. With an absolute positional accuracy of 4 mas, SIM will improve on the best currently available measures by 2 to 3 orders of magnitude, providing parallaxes accurate to $10 \%$ and transverse velocities accurate to $0.2 \mathrm{~km} / \mathrm{s}$ anywhere in the Galaxy, to stars as faint as 20 magnitude.

The following is a brief list of some of the important areas of astrophysics in which SIM will play an important role:

- Stellar Dynamics of the Galaxy. Using precision astrometry, SIM will address many fundamental questions concerning the mass distribution in our Galaxy, the dynamics of its stars, and the evolution of its stellar populations.

- Masses and Evolution of Stars in Close Binary Systems. By detecting the astrometric signature of the binary star orbit, SIM can determine the masses and orbits of a large number of ordinary binary star systems, as well as more exotic systems: white dwarf/CVs, neutron stars and black holes.

- Rotational Parallaxes. Distances to nearby spiral galaxies can be measured directly, without use of luminosity-based indicators, using proper motions of their brightest stars due to rotation of the disk about the center of the galaxy.

- Stellar Luminosities and Calibration of Distance Indicators. SIM will calibrate the luminosities of Cepheid and RR Lyrae 'standard candle' variable stars, and planetary nebulae. These sources are used as standard candles in distance determinations for calculating the Hubble constant, and in distance measurements to globular clusters.

- MACHO Gravitational Microlensing Events. SIM will be able to detect the astrometric signature of microlensing events caused by massive compact halo objects (MACHOs) along the line of sight [6]. Masses for these objects can be inferred, providing important clues to the nature of dark matter in the Galaxy.

- Ages of Globular Clusters. Stellar evolution models for clusters apparently conflict with the age of the universe inferred from the Hubble expansion. SIM will measure the distances to globular clusters directly using trigonometric parallax, and hence the luminosities of the oldest main-sequence stars can be determined. Subtle effects due to reddening and metallicity effects can be studied.

- Dynamics of Small Stellar Systems. By measuring the proper motions of globular clusters, the mass distribution of the Galaxy can be determined. This will help our understanding of the formation of the Galactic halo and the globular cluster system. Tidal tails from disrupted dwarf spheroidal galaxies [7] also provide a powerful means of tracing the Galactic potential. 
The work described in this paper was performed at the Jet Propulsion Laboratory, California Institute of Technology, under a contract with the National Aeronautics and Space Administration.

\section{References}

1. Unwin S.C., Shao M., Peterson D.M., Jones C.P. (1999) Space Interferometry Mission Science Requirements Document. JPL internal document

2. Mayor M., Queloz, D. (1995) A Jupiter-mass companion to a solar-type star. Nature 378, 355

3. Marcy G.W., Butler R.P. (1996) A planetary companion to 70-Virginis. ApJ 464, L147

4. Butler R.P., Marcy G.W., Fischer D.A., Brown T.W., Contos A.R., Korzennik S.G., Nisenson P., Noyes R.W. (1999) Evidence for Multiple Companions to Upsilon Andromedae. ApJ (submitted)

5. Peterson D. M. (1995) Scientific Support for Space Interferometry, Spaceborne Interferometry II. Proc. SPIE 2477, 2

6. Boden A., Shao M., Van Buren D. (1998) Astrometric Observation of MACHO Gravitational Microlensing. ApJ 502, 538

7. Ibata R.A., Wyse R.F.G., Gilmore G., Irwin M.J., Suntzeff N.B. (1997) The kinematics, orbit, and survival of the Sagittarius dwarf spheroidal galaxy. AJ 113,634 . 